\title{
Decreased maximal aerobic capacity with use of a triphasic oral contraceptive in highly active women: a randomised controlled trial
}

\author{
C M Lebrun, M A Petit, D C McKenzie, J E Taunton, J C Prior
}

See end of article for authors' affiliations

.....................

Correspondence to:

Dr Lebrun, Fowler Kennedy

Sport Medicine Clinic 3M

Centre, University of

Western Ontario, London,

ON, Canada N6A 3K7;

clebrun@uwo.ca

Accepted 25 August 2002
Br J Sports Med 2003;37:315-320

Background: Oral contraceptives are commonly used by women athletes. However, their effect on athletic performance is unclear.

Objectives: To examine the effects of a moderate dose, triphasic oral contraceptive on measures of athletic performance in highly trained women athletes.

Methods: This is a double blind, placebo controlled trial in 14 women with ovulatory menstrual cycles and maximal aerobic capacity $\left(\mathrm{VO}_{2} \mathrm{MAX}\right) \geqslant 50 \mathrm{ml} / \mathrm{kg} / \mathrm{min}$. Four measures of athletic performance were tested: $\mathrm{VO}_{2} \mathrm{MAX}$, anaerobic capacity (anaerobic speed test), aerobic endurance (time to fatigue at $90 \%$ of $\mathrm{VO}_{2} \mathrm{MAX}$ ), and isokinetic strength (Cybex II dynamometer). Height, weight, and six skinfold measurements were also recorded. All these observational tests were completed during both the follicular and mid-luteal phases of an ovulatory menstrual cycle. Cycle phases were confirmed by assaying plasma oestradiol and progesterone. Participants were subsequently randomly assigned to either a tricyclic oral contraceptive or placebo and retested in identical fashion (oral contraceptive phase).

Results: Absolute and relative changes in $\mathrm{VO}_{2} \mathrm{MAX}$ from follicular to oral contraceptive phase decreased in the oral contraceptive group by $4.7 \%$, whereas the placebo group showed a slight increase $(+1.5 \%)$ over the same time period. Two of the women taking oral contraceptive had decreases of 4 and 9 $\mathrm{ml} / \mathrm{kg} / \mathrm{min}$. In contrast, most women in the placebo group improved or maintained $\mathrm{VO}_{2} \mathrm{MAX}$. There was also a significant increase in the sum of skinfolds in women taking oral contraceptive compared with those taking placebo $(p<0.01)$. There were no significant changes in other physiological variables (maximum ventilation, heart rate, respiratory exchange ratio, packed cell volume) or measures of performance (anaerobic speed test, aerobic endurance, isokinetic strength) as a function of oral contraceptive treatment.

Conclusions: The decrease in $\mathrm{VO}_{2} \mathrm{MAX}$ that occurs when oral contraceptive is taken may influence elite sporting performance in some women. Further studies are required to determine the mechanisms of this change.
E te women athletes are prescribed oral contraceptives for a variety of purposes, including contraception, cycle regulation, control of dysmenorrhoea, and treatment of amenorrhoea. Compared with the first generation pills, newer, moderate dose, triphasic formulations have fewer side effects such as weight gain, fluid retention, and alterations in lipid profiles. ${ }^{12}$ However, even these moderate dose oral contraceptive formulations have ethinyl oestradiol levels that are 3-5 times the oestrogen equivalent of endogenous oestradiol, and norethindrone levels that are $1-2$ times (spread over 21 days) higher progestin levels than endogenous progesterone. ${ }^{3}$ The effects of these high levels of exogenous hormones on muscle strength, aerobic capacity, and athletic performance are not known.

A few controlled trials have examined effects of oral contraceptive on indicators of exercise performance. A wide diversity in the oestrogen and progestin components of the oral contraceptive, the range of fitness parameters assessed, and varying fitness levels of the participants make the studies difficult to interpret. Results were mixed, showing changes in substrate metabolism, improved running economy, ${ }^{4}$ decreased $\mathrm{Vo}_{2} \mathrm{MAX}^{56}$ in conjunction with a significant reduction in mitochondrial citrate, or no significant effects of oral contraceptive on performance. ${ }^{7-9}$ Overall, there is a lack of consensus about the effects of oral contraceptives on athletic performance. ${ }^{10}{ }^{11}$ To our knowledge, there are no randomised studies of the effects of oral contraceptive on performance in highly trained athletes.
We previously reported observational changes in aerobic performance across the menstrual cycle in 16 highly trained female athletes. ${ }^{12}$ As an extension of that study, the same women were subsequently randomised to oral contraceptive or placebo for two months, and retested between days 14 and 17 of the second cycle. The purpose of this randomised double blind, placebo controlled study was to examine characteristics of athletic performance with oral contraceptive use in highly active women.

\section{METHODS AND MATERIALS \\ Subjects}

Ethical approval was obtained from the committee on human experimentation of the University of British Columbia, and all participants gave written informed consent. Athletic women aged 18-40 were recruited. Women were initially excluded if they did not meet the following criteria: (a) regular menstrual cycles (24-35 days in length); (b) no oral contraceptive use in the three months before entering the study; $(c)$ participating on a regular basis in competitive aerobic activity-that is, running, cycling, triathlon, rowing, cross country skiing.

At an initial screening session, $\mathrm{VO}_{2} \mathrm{MAX}$ and general health history were assessed. Volunteers who had $\mathrm{Vo}_{2} \mathrm{MAX}$ values less than $50 \mathrm{ml} / \mathrm{kg} / \mathrm{min}$ were excluded. Women were further excluded if they had any potential risk factors for oral contraceptive administration including smoking, any significant past medical condition, or were taking any medication that 


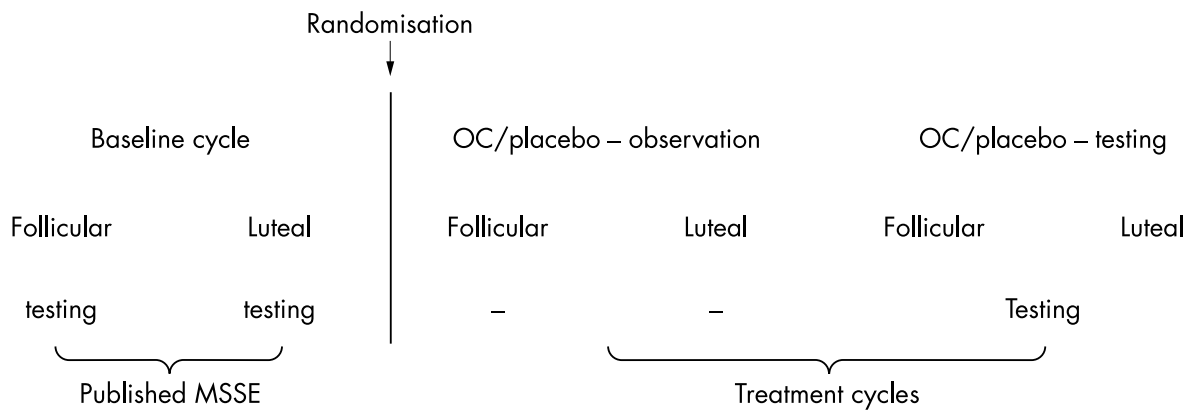

Figure 1 Study design showing three cycles with testing twice in the first cycle and once during the second treatment cycle. OC, Oral contraceptive; MSSE, Medicine and Science in Sports and Excercise. ${ }^{12}$

might interfere with exercise testing or administration of oral contraceptive. Participants were also required to have a physical examination, including a pelvic examination and Pap smear, carried out by their own doctor. Women taking supplements or iron were asked to maintain the exact dosage throughout the entire length of the three cycle study (of which this controlled trial was the third cycle).

Of the 51 volunteers for the initial study, ${ }^{12} 17$ met all criteria and completed all three tests (follicular, luteal, and oral contraceptive or placebo). Three of these 17 women did not show hormonal evidence of ovulation during the observational study, and their test results were subsequently excluded from the analyses. Data are presented for 14 women who completed this controlled trial.

\section{Study design}

Figure 1 presents an overview of the study design and testing protocol. Testing was performed during both the early follicular (days 3-8) and mid-luteal (days 4-9 after "ovulation") phases of an ovulatory menstrual cycle. A resting level of serum progesterone higher than $16 \mathrm{nmol} / \mathrm{l}$ was required to confirm ovulation. ${ }^{13}$ After follicular and luteal phase tests, participants were randomly assigned to either an oral contraceptive $(n=7)$ or placebo $(n=7)$ group. Participants and investigators were blind to group assignment.

A triphasic oral contraceptive (Synphasic; Pharmacia Canada, Mississanga, Ontario, Canada) was used. Synphasic contains a constant concentration of $0.035 \mathrm{mg}$ ethinyl oestradiol and two different doses of norethindrone in three phases (days 1-7, $0.5 \mathrm{mg}$; days 8-16, $1.0 \mathrm{mg}$; and days 17-21, $0.5 \mathrm{mg}$ ) equalling a total norethindrone dose of $15.0 \mathrm{mg}$ over the 21 day cycle. All women took an unmarked lactose capsule containing either oral contraceptive or placebo following the recommended schedule of daily administration for three consecutive weeks and stopping for one week. Capsules were taken as above for two consecutive months. The treatment testing session was performed between days 14 and 17 (mean (SD) 14.4 (0.5) days) of the second cycle of oral contraceptive or placebo administration (fig 1). These days were chosen as these were the last days of the highest progestin dose of the triphasic oral contraceptive.

\section{Testing protocol}

All tests were completed on two successive days during each phase (follicular, luteal, and treatment). Because of the double blind nature of the study, treatment phase tests were conducted on days 14-17 of the cycle rather than based on day of ovulation. Although this may theoretically be during the luteal phase in the subjects on placebo, there was a high degree of individual variability in the actual day of ovulation. Most women were actually in the mid- or late-follicular phase according to serum progesterone levels. Two women in each group appeared to have ovulated before oral contraceptive treatment tests.
For the first day, participants reported to the laboratory in a fasted, resting state, and venous blood samples, $\mathrm{VO}_{2} \mathrm{MAX}$, and anaerobic performance were assessed. Measurements of isokinetic strength, aerobic endurance, and assessment of body composition were completed on the second day of testing.

\section{Anthropomentry and body composition}

Height and weight (Detecto industrial scale) were measured to the nearest $0.1 \mathrm{~cm}$ and $0.1 \mathrm{~kg}$ respectively. Skinfold thickness was measured at six sites (biceps, triceps, subscapular, suprailiac, anterior thigh, and medial calf) with a Harpenden skinfold caliper (John Bull, UK British Indicators Ltd, St Albans, Herts, UK). Skinfold measurements are reported as the sum of all values. Percentage body fat was assessed by underwater densitometry and calculated using the Siri formula. ${ }^{14}$

\section{Blood samples}

Blood samples ( $15 \mathrm{ml}$ ) were obtained by venepuncture. They were kept cool (in an ice/water bath), and processed when testing was completed. One tube was taken to the laboratory at the University Hospital for determination of an automated blood count (CoulterS + STKR). The remaining blood was spun in a refrigerated centrifuge (Damon/IEC Clini-Cool) for 10 minutes at $3000 \mathrm{rpm}$. Plasma was stored in Venoject plain silicone coated glass tubes at $-20^{\circ} \mathrm{C}$ until analysis using commercially available no extraction, solid phase ${ }^{125} \mathrm{I}$ radioimmunoassays (Coat-A-Count Estradiol and Coat-A-Count Progesterone; Diagnostic Products Corporation). Over the duration of the study, blood samples were coded and analysed in three separate batches by an independent observer. All samples from one woman were analysed together, and at least one participant from each of the two experimental groups was included in each assay. Intra-assay coefficients of variation were $10.6 \%$ for oestradiol and $10.3 \%$ for progesterone. Interassay coefficients of variation ranged from 4.2 to $8.1 \%$ for oestradiol and from 7.2 to $10.0 \%$ for progesterone (Diagnostic Products Corporation, Los Angeles, California, USA). Assay sensitivities were $2.9 \mathrm{pmol} / \mathrm{l}$ for oestradiol and $0.16 \mathrm{nmol} / \mathrm{l}$ for progesterone. The oestradiol assay does not detect the ethinyl oestradiol and the progesterone assay does not detect the norethindrone in this oral contraceptive.

\section{Aerobic capacity}

$\mathrm{VO}_{2} \mathrm{MAX}$ was assessed using a standard running protocol. ${ }^{15}$ After a 10 minute warm up at a self selected pace (between 2.2 and $2.7 \mathrm{~m} / \mathrm{s}$ ), a continuous progressive workload was carried out on a level grade, beginning at a speed of $2.2 \mathrm{~m} / \mathrm{s}$, and increasing by $0.22 \mathrm{~m} / \mathrm{s}$ each minute until fatigue. Heart rate was monitored using a Polar Vantage heart rate monitor and recorded at 45 seconds into each stage. Expired gases were continuously sampled and analysed using a Beckman Metabolic Measurement Cart (OM-11 oxygen analyser and 


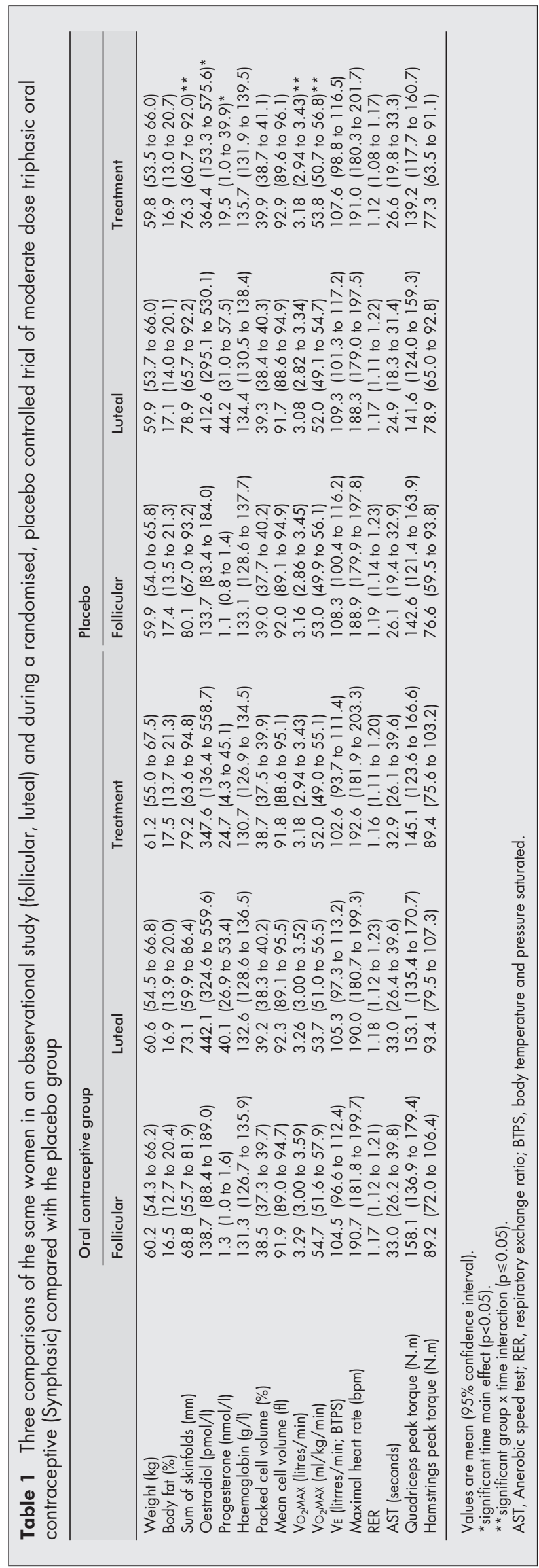

LB-2 carbon dioxide analyser), and tabulated by a data acquisition system (Hewlett-Packard 3052A) which determined respiratory gas exchange variables every 15 seconds. The volume transducer was calibrated using a 1.0 litre syringe, and both gas analysers were calibrated with standardised calibration gases and room air before each test. A maximal test was defined by achievement of at least two of the following three criteria: a plateau or decrease in $\mathrm{Vo}_{2} \mathrm{MAX}$ despite an increase in work load; a respiratory exchange ratio greater than or equal to 1.1 ; attainment of at least $90 \%$ of predicted maximum heart rate.

\section{Endurance performance}

Endurance performance was assessed as the running time in seconds to fatigue at a treadmill velocity requiring about $90 \%$ of $\mathrm{VO}_{2} \mathrm{MAX} .{ }^{16}$ This workload was determined by taking $90 \%$ of the treadmill speed at which the subject completed her last full minute of running before stopping the initial (observational, follicular phase) $\mathrm{Vo}_{2} \max$ test. The workload speed remained constant for the next two testing sessions, regardless of any subsequent variations in the actual $V_{O_{2}} \operatorname{MAX}$ measurement.

\section{Anaerobic performance}

High intensity running performance was assessed by the anaerobic speed test (AST). ${ }^{17}$ Participants rested for at least 1.5 hours after the $\mathrm{VO}_{2} \operatorname{MAX}$ test before measurement of their anaerobic performance. After an adequate warm up, subjects performed the run at $8 \mathrm{mph}(3.52 \mathrm{~m} / \mathrm{s})$ at a $20 \%$ incline until fatigue (defined as an inability of the subject to continue at the set treadmill speed). Time (seconds) to fatigue was used as the performance index. The test-retest reliability of the AST has been documented $(r=0.76-0.91) .{ }^{16}$

\section{Isokinetic strength}

Isokinetic strength was measured on a Cybex II dynamometer. Participants were positioned on the Cybex table with the lateral femoral condyle aligned with the dynamometer axis of rotation. After a short warm up at a velocity of 240 degrees/s, peak torque (N.m) for knee flexion and extension was recorded at a velocity of 30 degrees/s. For each leg, the best value of three different attempts was recorded, and the average of both legs reported. The coefficient of variation for this protocol is $5.9 \%{ }^{16}$

\section{Statistical analysis}

Repeated measures analysis of variance was used to test for main effects and interactions of group (oral contraceptive or placebo) and phase (follicular, luteal, treatment). Dependent variables included absolute and relative $\mathrm{VO}_{2} \mathrm{MAX}$, anaerobic capacity (AST), isokinetic strength (Cybex II measurement of peak torque of knee flexion and extension), and aerobic endurance (time to exhaustion at $90 \%$ of $\mathrm{Vo}_{2} \mathrm{MAX}$ ). Weight, sum of skin folds, percentage body fat, concentration of oestradiol, progesterone, and haemoglobin, and packed cell volume were also analysed for changes over time as a result of either phase or treatment protocol. Data were analysed using SPSS (version 10.1, 2000; SPSS Inc, Chicago, Illinois, USA). $p<0.05$ was considered significant.

Although all tests during the controlled oral contraceptive treatment were performed during days 14-17 of the cycle, for all but four women (two in each group), progesterone levels were $<16 \mathrm{nmol} / \mathrm{l}$ in the treatment phase. To illustrate group differences in figures and when reporting percentage change, oral contraceptive treatment tests were compared with observational follicular phase tests. However, for the four women who from progesterone levels appeared to have ovulated, tests were compared with their own previous observational luteal phase tests. 


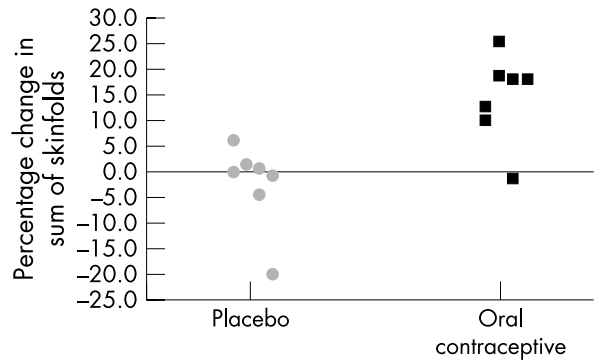

Figure 2 Percentage change in sum of skinfolds from follicular to treatment phase for women in the placebo and oral contraceptive groups.

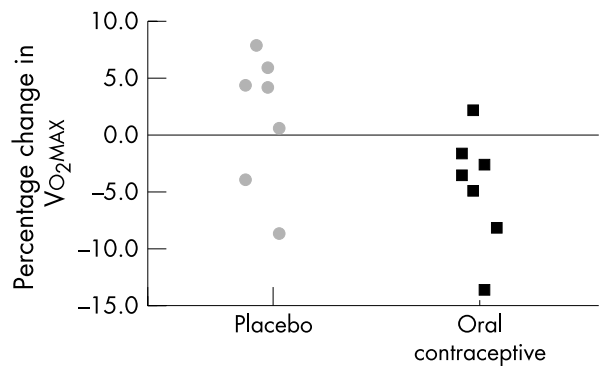

Figure 3 Percentage change in $\mathrm{VO}_{2} \mathrm{MAX}$ from follicular to treatment phase for women in the placebo and oral contraceptive groups.

\section{RESULTS}

Table 1 presents characteristics for each group in the three phases: follicular phase, luteal phase, and oral contraceptive treatment period. Follicular phase values were not affected by random assignment to oral contraceptive or placebo.

\section{Body composition}

Body weight and percentage fat by underwater weighing were similar in the two groups at each phase. Subjects in the oral contraceptive group increased weight $(+1 \mathrm{~kg})$ and percentage fat by underwater weighing $(+1 \%)$ from the follicular to the treatment phase, but those in the placebo group showed no change. Group differences were not significant (group $\times$ phase, $p>0.05)$. Change in sum of skinfolds was significantly different between the groups over time, with a larger increase in the oral contraceptive group (group $\times$ phase, $\mathrm{p}=0.004$; fig 2).

\section{Hormones and blood samples}

As expected, oestradiol $(\mathrm{p}<0.01)$ and progesterone $(\mathrm{p}<0.01)$ values differed significantly between the follicular, luteal, and treatment tests for all subjects, but did not vary as a function of oral contraceptive treatment (table 1). Haemoglobin concentration, packed cell volume, and mean red cell volume did not differ significantly between groups or within each group over time.

\section{Exercise performance}

Aerobic capacity

Both absolute and relative $\mathrm{VO}_{2} \mathrm{MAx}$ differed between the oral contraceptive and placebo groups over time $(\mathrm{p}=0.050$ and $\mathrm{p}$ $=0.019$ respectively). Aerobic capacity decreased in the oral contraceptive group across the three phases. In contrast, there was a slight decrease in $\mathrm{VO}_{2} \mathrm{MAX}$ between the follicular and luteal phase tests, but an increase in the third test in the placebo group. The mean decrease from the follicular to treatment phase was $4.7 \%$ in the oral contraceptive group compared with a $1.5 \%$ improvement with placebo. Figure 3 illustrates individual changes. There were no significant fluctuations in maximum minute ventilation ( $\mathrm{VE})$, maximum heart rate, or maximum respiratory exchange ratio accompanying changes in $\mathrm{VO}_{2} \mathrm{MAX}$.

Endurance performance, anaerobic capacity, and strength

There were no significant differences in endurance performance (at $90 \%$ of $\mathrm{VO}_{2} \mathrm{MAX}$ ), anaerobic capacity (as measured by the AST), or isokinetic strength (on a Cybex II dynamometer) within or between groups over time (table 1 ).

\section{DISCUSSION}

To our knowledge, this is the first randomised, double blind, placebo controlled trial of the effects of oral contraceptive on indicators of athletic performance in highly trained women $\left(\mathrm{VO}_{2} \mathrm{MAX}=50 \mathrm{ml} / \mathrm{kg} / \mathrm{min}\right)$. Use of moderate dose triphasic oral contraceptive resulted in a mean decrease in $\mathrm{VO}_{2} \mathrm{MAX}$ of $4.7 \%$ in trained women compared with a $1.5 \%$ improvement with placebo. Although there was high individual variability in response to oral contraceptive administration, there was a clear trend towards a detriment in $\mathrm{VO}_{2} \mathrm{MAX}$ in highly trained women taking oral contraceptive. The decrease in $\mathrm{Vo}_{2} \mathrm{MAX}$ was accompanied by an increase in sum of skinfolds, but not by significant changes in weight or measures of strength, anaerobic, or endurance performance.

Previous studies on the role of oral contraceptive on athletic performance have assessed a variety of performance indicators with varying protocols, duration and type of oral contraceptive use, and fitness levels of participants. ${ }^{10}{ }^{11}$ Researchers have examined the effect of oral contraceptive on $\mathrm{VO}_{2} \mathrm{MAX}$, submaximal endurance performance, strength, anaerobic capacity, and side effects such as weight gain.

\section{Maximal aerobic capacity}

Studies in untrained or moderately trained women have shown mixed effects of oral contraceptive use on $\mathrm{VO}_{2} \mathrm{MAX}$. Daggett and colleagues ${ }^{5}$ showed a significant reduction in $\mathrm{VO}_{2} \mathrm{MAX}$ (from 44.6 to $39.8 \mathrm{ml} / \mathrm{kg} / \mathrm{min}$ ) in a group of seven moderately trained women after one to two months of oral contraceptive use. Another study ${ }^{6}$ used a design similar to the present investigation, with both a control group $(n=6)$ and an oral contraceptive group $(n=6)$. Over six months, the control group increased aerobic capacity by about $8 \%$ (from $42.6(2.8)$ to 45.9 (5.8) $\mathrm{ml} / \mathrm{kg} / \mathrm{min}$ ), whereas after six months of monophasic oral contraceptive administration, $\mathrm{Vo}_{2} \mathrm{MAx}$ had decreased by about $7 \%$ (from 41.2 (11.8) to 38.4 (9.8) $\mathrm{ml} / \mathrm{kg} / \mathrm{min}$ ). Changes were associated with a decrease in the oxygen pulse (12.1 (3.2) to 11.2 (2.2) ml per beat) and were reversible on discontinuation of treatment. ${ }^{6}$ The smaller differences in change in $\mathrm{VO}_{2} \mathrm{MAX}$ in our study could be due to lower doses of exogenous steroids in the oral contraceptive, shorter duration of administration, or the greater fitness level of our participants. In contrast, other studies showed no effect of oral contraceptives on $\mathrm{VO}_{2} \mathrm{MAX}$ in moderately trained women. ${ }^{18}$

As in our study, the observed decreases in $\mathrm{VO}_{2} \mathrm{MAx}$ have not been directly linked to significant alterations in $\mathrm{O}_{2}$ carrying capacity of the blood (haemoglobin concentration or packed cell volume), or other physiological measurements influencing $\mathrm{O}_{2}$ uptake or delivery to the tissue. Although the mechanisms are unclear, exogenous oestrogen may exert a deleterious effect on aerobic capacity. The changes are probably not due to progestin. A well controlled, randomised, double blind, placebo controlled trial showed that high doses of medroxyprogesterone (20 mg three times/day for five doses) in men had no effect on $\mathrm{VO}_{2} \mathrm{MAx}$, but did increase minute ventilation as expected. ${ }^{19}$

\section{Submaximal endurance performance}

Oral contraceptives have been shown to alter substrate metabolism, including carbohydrates and lipids, ${ }^{20}{ }^{21}$ and to cause a decrease in blood glucose with heavy exercise. ${ }^{21}$ These 
actions, which may be linked to high oestrogen, ${ }^{22}$ could decrease endurance performance by reducing the fuel available for exercise. In our study, there were no significant differences between oral contraceptive and placebo groups in their performance on the endurance run. In contrast, a recent study reported improved submaximal running economy with oral contraceptive use. ${ }^{4}$ These differences may be partially explained by the type of tests used to assess endurance performance. In our study, participants ran at $90 \%$ of $\mathrm{VO}_{2} \mathrm{MAX}$ until fatigue. Giacomoni and colleagues ${ }^{4}$ assessed running economy at 7,8 , and $9 \mathrm{~km} / \mathrm{h}$, equal to about $60-80 \% \mathrm{VO}_{2} \mathrm{MAx}$. In that study, differences in running economy tended to be greater at lower intensities ( 7 and $8 \mathrm{~km} / \mathrm{h} v 9 \mathrm{~km} / \mathrm{h}$ ). There may be a different response to oral contraceptive use at lower compared with higher intensities of exercise.

\section{Strength and anaerobic capacity}

It has been postulated that oral contraceptive use may prevent normal decreases in strength that occur during the luteal phase of a menstrual cycle. ${ }^{23}$ We saw no significant differences in measurements of muscle strength with oral contraceptive use or placebo. The overall effect of oral contraceptive on strength remains unknown.

We also saw no changes in anaerobic performance as a function of oral contraceptive use. Although few studies have been performed, there does not appear to be any effect of oral contraceptive on energy metabolism for short term anaerobic work. However, the scores for this test were relatively low in most of the athletes in our study, indicating a low anaerobic capacity. This is consistent with the fact that these subjects were well trained for predominantly aerobic-type activities. The effect of oral contraceptive use on anaerobic performance in highly trained anaerobic athletes should be explored.

\section{Benefits and side effects of oral contraceptives}

Potential benefits of oral contraceptive for athletic performance include decreased dysmenorrhoea, iron deficiency, and anaemia risks in women experiencing heavy flow. All participants had normal haemoglobin levels, and there were no significant oral contraceptive related changes in either haemoglobin or packed cell volume over six weeks. This is in agreement with other short term studies. ${ }^{64}$

Early studies of high dose oral contraceptive formulations documented side effects, including fluid retention and weight gain. Biphasic and triphasic pills contain 30-40\% lower levels of hormone and appear to have a corresponding decrease in adverse effects. ${ }^{25}{ }^{26}$ In this study, athletes taking oral contraceptive had a non-significant weight gain of about $1 \mathrm{~kg}$ over six weeks compared with women in the placebo group. The significant increase in sum of skinfolds in the oral contraceptive group suggests that the weight increase was due primarily to an increase in subcutaneous body fat, although increased water retention cannot be excluded. Overall, recent noncontrolled studies suggest no long term change in weight with oral contraceptive use, ${ }^{26}$ but changes in body composition have not been assessed.

\section{Limitations}

A caveat in this type of research (that only became evident during the course of the study) is the relative impossibility of carrying out true double blind studies with oral contraceptives. Approximately half of the participants were aware that they were taking oral contraceptives because of subtle changes, alterations in the pattern of their normal menstrual cycles, and the presence of side effects such as breakthrough bleeding throughout the cycle. Also, as noted, two of the seven women on the moderate dose oral contraceptive actually still showed hormonal evidence of ovulation.

Studies of this nature are also difficult to control because of the tremendous individual variability in timing of ovulation,

\section{Take home message}

This randomised, double blind, placebo controlled study showed that oral contraceptive use may decrease aerobic capacity in some highly trained athletes, but there is high individual variability. Further study is needed to determine mechanisms of change and to document whether changes are reversible or persist with longer term use.

response to oral contraceptive treatment, and cycle phase. Although we meticulously performed all treatment phase tests on days 14-17 of the menstrual cycle, there was a high degree of individual variability in the actual day of ovulation in the test cycle. Most women were actually in the mid- or late-follicular phase according to serum progesterone levels. However, two women in each group appeared to have ovulated before treatment tests. Results of our study were similar regardless of the phase with which data were compared. Nonetheless, the difficulty of repeating tests in the exact phase of the cycle over time should be noted.

\section{Summary}

The results of this study indicate that administration of this moderate dose triphasic oral contraceptive for two cycles does not have any apparent or measurable effect on most components of athletic performance in this group of elite women athletes. The small decreases in $\mathrm{VO}_{2} \operatorname{MAx}$ that occurred in women taking oral contraceptive suggest that exogenous oestrogen may exert a deleterious effect on aerobic capacity with potential implications for elite performance. The magnitude of this effect varied between individuals. Further studies are necessary to delineate potential mechanisms of the change in functional aerobic capacity and body weight, and to further document whether changes in performance are reversible on discontinuation of treatment. Studies of the effects of oral contraceptive after longer term use (more than six months), and with larger sample size will also provide important insight into the effects of oral contraceptives on athletic performance. In addition, different formulations of oral contraceptive should be compared.

\section{AUTHORS' NOTE}

Since acceptance of our paper, we have learned that another group of authors has published similar findings from their work: Casazza GA, Suh S-H, Miller BF, et al. Effects of oral contraceptives on peak capacity. J Appl Physiol 2002;93:1698-1702

\section{Authors' affiliations}

C M Lebrun, M A Petit, D C McKenzie, J E Taunton, J C Prior, Fowler Kennedy Sport Medicine Clinic $3 M$ Centre, University of Western

Ontario, London, ON N6A 3K7, Canada

\section{REFERENCES}

1 Collins DC. Sex hormone receptor binding, progestin selectivity, and the new oral contraceptives. Am J Obstet Gynecol 1994;170:1508-13.

2 Coney P, Washenik K, Langley RG, et al. Weight change and adverse event incidence with a low-dose oral contraceptive: two randomised placebo-controlled trials. Contraception 2001;63:297-302.

3 Goodman AG, Goodman LS, Gilman A. The pharmacological basis of therapeutics. New York: MacMillian, 1980.

4 Giacomoni $M$, Falgairette $G$. Decreased submaximal oxygen uptake during short duration oral contraceptive use: a randomised cross-over trial in premenopausal women. Ergonomics 2000;43:1559-70.

5 Daggett A, Davies B, Boobis L. Physiological and biochemical responses to exercise following oral contraceptive use[abstract]. Med Sci Sports Exerc 1983;15:174.

6 Notelovitz M, Zauner C, McKenzie L, et al. The effect of low-dose contraceptives on cardiorespiratory function, coagulation, and lipids in exercising young women: a preliminary report. Am J Obstet Gynecol 1987;156:591-8.

7 Huisveld IA, Hospers JEH, Bernink M, et al. The effect of oral contraceptives and exercise on hemostatic and fibrinolytic mechanisms in trained women. Int J Sports Med 1983;4:97-103. 
8 McNeill AW, Mozingo E. Changes in the metabolic cost of standardized work associated with the use of an oral contraceptive. J Sports Med $1981 ; 21: 238-44$.

9 Lynch NJ, Nimmo MA. Effects of menstrual cycle phase and oral contraceptive use on intermittent exercise. Eur J Appl Physiol 1998:78:565-72

10 Lebrun CM. Effect of the different phases of the menstrual cycle and oral contraceptives on athletic performance. Sports Med 1993;16:400-30.

11 Frankovich RJ, Lebrun CM. The athletic woman: menstrual cycle, contraception and performance. Clin Sports Med 2000;1 9:251-71.

12 Lebrun CM, McKenzie DC, Prior JC, et al. Effects of menstrual cycle phase on athletic performance. Med Sci Sports Exerc 1995;27:437-44.

13 Abraham GE, Maroulis GB, Marshall JB. Evaluation of ovulation and corpus luteum functioning using measurements of plasma progesterone. Obstet Gynecol 1974;44:522-5.

14 Siri WE. Body composition from fluid spaces and density analysis of methods. In: Brozek J, Henschel A, eds. Techniques for measuring body composition. Washington, DC: National Academy of Science and National Research Council, 1961

15 Parkhouse WS, McKenzie DC, Hochachka PW, et al. Buffering capacity of deproteinized human vastus lateralis muscle. $J$ Appl Physiol 1985;58:14-17

16 MacDougall JD, Wenger HA, Green HA, eds. Physiological testing of the high-performance athlete. 2nd ed. Champaign, IL: Human Kinetics Publishers, 1991.
17 Cunningham DA, Faulkner JA. The effect of training on aerobic and anaerobic metabolism during a short exhaustive run. Med Sci Sports Exerc 1969;1:65-9.

18 Bryner R, Toffle R, Ullrich I, et al. Effect of low dose oral contraceptives on exercise performance. Br J Sports Med 1996;30:36-40.

19 Bonekat H, Dombovy M, Staats B. Progesterone-induced changes in exercise performance and ventilatory responses. Med Sci Sports Exerc 1987; 19:118-23.

20 Beck P. Contraceptive steroids: modification of carbohydrate and lipid metabolism. Metabolism 1973;22:841-55.

21 Bonen A, Haynes FW, Graham TE. Substrate and hormonal responses to exercise in women using oral contraceptives. J Appl Physiol 1991;70:1917-27.

22 Ashley CD, Kramer ML, Bishop P. Estrogen and substrate metabolism: a review of contradictory research. Sports Med 2000;29:221-7.

23 Petrofsky JS, LeDonne DM, Rinehart JS, et al. Isometric strength and endurance during the menstrual cycle. Eur J Appl Phsyiol 1976;35:1-10

24 Fortney SM, Beckett WS, Carpenter AJ, et al. Changes in plasma volume during bed rest: effects of the menstrual cycle and estrogen administration. J Appl Physiol 1988;65:525-33.

25 Greenblatt RB. Oral contraceptives: the state of the art. Clin Ther 1985;8:6-27.

26 Rosenberg $M$. Weight change with oral contraceptive use and during the menstrual cycle. Contraception 1998;58:345-9.

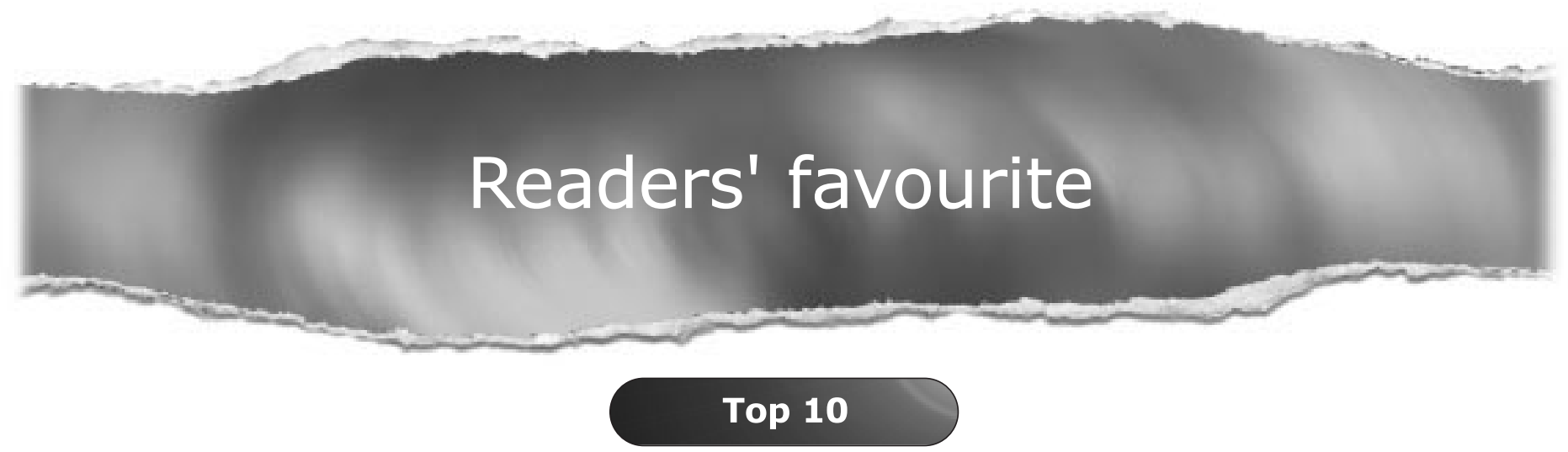

Click on the "Top 10" button on the homepage to see which are the best read articles each month

www.bjsportmed.com 